\title{
The $\lambda$-Characteristics of the Ring Laser Based on Non-Uniform SOA
}

\section{Zhi Wang, Limei Zhang, Yingfeng Liu, Lanlan Liu}

Institute of Optical Information, Beijing Jiaotong University, Beijing 100044 Key Laboratory of Luminescence and Optical Information, Ministry of Education, China.

Email: zhiwang@bjtu.edu.cn

Received October 2013

\begin{abstract}
Semiconductor optical amplifier-based ring cavity laser (SOA-RL), which has been widely used in optical communications, optical fiber sensing, and bio-photonics fields, can be tuned at an ultra high speed up to Mega Hertz over $100 \mathrm{~nm}$ bandwidth range with high SNR and flatness output. A steady-state model and segmentation algorithms are employed to investigate the gain spectra of four kinds of non-uniform SOA and the lasing wavelength of the SOA-RL. It shows that the dependence of the lasing wavelength on the average width is stronger when the light propagates from narrower to wider end than conversely, and there are some particular structures to show ultra high stability lasing wavelength. It is supposed that the main reason could be the carrier density distribution along the propagation.
\end{abstract}

Keywords: Semiconductor Optical Amplifier; Non-Uniform; Ring Laser; Gain

\section{Introduction}

Ring laser based on semiconductor optical amplifier (SOA-RL) $[1,2]$ can be conveniently tuned as wavelength swept laser or Fourier domain mode locked laser [3], and employed in many fields, such as the characteristic test for optical fiber communication system [4], optical coherence tomography (OCT) [5], ultra high resolution distributed optical fiber sensors, optical power equilibrium [6] and all optical packet switching [7]. The SOA-RL shows us some properties of both the ring cavity and external cavity lasers, and can operate at some different regimes with some in-line devices, such as the filter, fiber coupler, isolator and polarization controller [8]. It is more easily to oscillate with single longitudinal mode and output narrow pulse sequence at high repeat rate with the structure of ring cavity [9]. SOA-RL has good compatibility with optical fiber systems, which can reduce kinds of connection loss and disturbance, such as the refraction loss, back scattering loss, and suppress the effect of the backward light into the SOA [10]. The flexibility and fiber-compatibility of the SOA-RL itself emphasize its importance in the future photonic devices and next generation optical telecommunications.

There are only a few literatures about the mechanism of the SOA-RL while lots of applications reports. The SOA shows particular properties with non-uniform injection [11], but there is no literature to discuss the SOA-RL with non-uniform injection. In fact, the non-uniform injection can be only realized for the SOA with non-uniform active layer, so the non-uniform SOA, instead of the non-uniform injection, will be investigated in this manuscript with segmentation method.

The gain properties of a few kinds of non-uniform SOAs are simulated and followed by the output of the SOA-RLs with the corresponding non-uniform SOAs.

\section{Model and Algorithm}

Figure 1 is the basic structure of the SOA-RL, in which the SOA (InPhenix Inc.) is the gain medium, the tunable filter (@1550 nm, FFP-TF2, Micron Optics Inc.) is driven by external signal (sawtooth or sinusoidal), the coupler is for feedback and output, isolators (ISO), polariza-

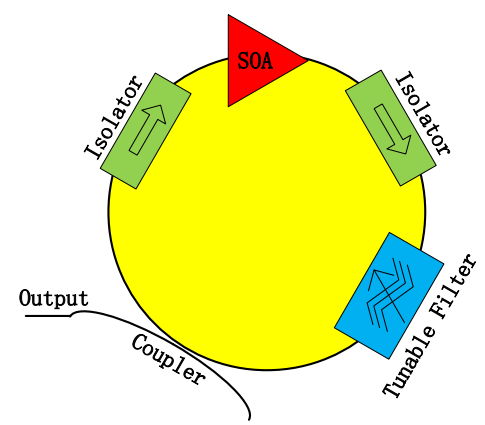

Figure 1. The basic scheme of the SOA-RL. 
tion control (PC), and dispersion management fibers are also included in the cavity.

The cavity length of the SOA-RL is about tens of meters or kilometers, the roundtrip time of light in the cavity is about hundreds of ns or $\mu \mathrm{s}$. The experiments show that tens of roundtrips is necessary to build up the laser from ASE with the amplifications by the SOA, and it will cost about a few $\mu$ s or ms, which is much longer than the gain recovery time of bulk SOAs (about hundreds of ps and dominated by the carrier recovery time), so the SOA can be regarded to operate at steady state.

The SOA is split into sections, and carrier density and photon density are regarded as constant in every section. The gain is wavelength (frequency) dependent, and the ASE band width is over $100 \mathrm{~nm}$, so the ASE photons is assumed to exist only at some discrete frequencies corresponding to integer multiples of cavity resonances, and the gain properties at other frequencies are obtained by fitting and interpolation.

For non-uniform SOA, the height $h(z)$ and the width $W(z)$ of the active layer will be $z$-dependent, so the rate equation of the carrier density $N$ at $z$ can be written as Equation (1) refer to Ref.[12].

$$
\begin{aligned}
& \frac{d N(z)}{d t}=\frac{I}{e F(z)}-R(N(z)) \\
& -\frac{\Gamma}{h(z) W(z)}\left\{\sum_{k=1}^{N_{s}} g_{m}\left(v_{k}, N(z)\right)\left(N_{s k}^{+}(z)+N_{s k}^{-}(z)\right)\right\}, \\
& -\frac{2 \Gamma}{h(z) W(z)}\left\{\sum_{j=1}^{N_{m}-1} g_{m}\left(v_{j}, N(z)\right) K_{j}\left(N_{j}^{+}(z)+N_{j}^{-}(z)\right)\right\}
\end{aligned}
$$

where $I$ is the amplifier bias current, $e$ is the electron charge, $L$ is the length of the active region, $\Gamma$ is the fraction of amplified photons resides in the active region, $g_{m}$ is the material gain coefficient. The bias current is assumed to pass through the active region only and have a uniform distribution across the active region width. The first term on the right hand side represents the addition of carriers to the active region from the bias current. These injected carriers are then depleted by various mechanisms occurring within the amplifier. $R(N)$ is the recombination rate term including both radiative and nonradiative carrier recombination rates. The third and fourth terms represent radiative recombination of carriers due to the amplified signal $\left(v_{k}\right)$ and amplified spontaneous emission (ASE, $v_{j}$ ), superscript “+” or “-” means forward or backward propagation light.

In Equation (1), $F(z)=h^{2}(z) \int_{0}^{L} W(z) / h(z) \mathrm{d} z$, could be called as volume factor. For the uniform SOA, the $F(z)$ is exactly the same as the volume of the active area, and even for the non-uniform SOA, when the height is uniform, $F(z)$ is also exactly the volume of the active area.

Considering the ring structure of the SOA-RL, the al- gorithm segmentation method for the quasi-steady state SOA comes from M. J. Connelly [12], we will not discuss the algorithm itself.

\section{Non-Uniform SOA}

In this manuscript, the non-uniform SOA has only the width of the active area z-dependent, which is shown in Figure 2. The SOA is segmented into 40 subsections for the whole length 700 micron, all the parameters, such as the size of the active area, the photon density, the carrier density, are considered as uniform in every particular section.

Equations (2a) to (2d)express the dependence of the width $W(z)$ on the position $z$, which are linear, triangular, exponential, and quadratic, where $N_{z}$ is the total section number, $W_{\text {ave }}$ is the average width along the propagation, $a$ and $b$ are parameters for different functions.

$$
\begin{gathered}
W_{0}(z)=a_{0} z+b_{0}, W_{\text {0ave }}=\frac{1}{2} a_{0} N_{z}+b_{0}, \\
W_{1}=\left\{\begin{array}{l}
a_{1} z+b_{1} \\
a_{1}\left(N_{z}-z\right)+b_{1}
\end{array}, W_{1 \text { ave }}=\frac{1}{4} a_{1} N_{z}+b_{1},\right. \\
W_{2}=b_{2} \exp \left(a_{2} z\right), W_{2 a v e}=\frac{b_{2}}{a_{2} N_{z}}\left(e^{a_{2} N_{z}}-1\right), \\
W_{3}=a_{3} z^{2}+b_{3}, W_{3 a v e}=\frac{1}{3} a_{3} N_{z}^{2}+b_{3} .
\end{gathered}
$$

Figure 3(a) shows the dependence of the width on the position for $W_{\text {ave }}=500 \mathrm{~nm}$ and $b=0.2 W_{\text {ave }}$. In order to check the gain property of the non-uniform SOA, the cw light inject from the narrower end and propagate to the wider end, or from the wider end to the narrower end. Figure 3(b) is the gain spectra for different non-uniform SOA when the input power is $-20 \mathrm{dBm}$, the legend with " $-c$ " means light propagating conversely from wider end to narrower end. It is very interesting that the gain with " $-c$ " is a little greater than that without " $-\mathrm{c}$ ", which means that the gain is higher when the light propagates from wider to narrower end, and the gain for uniform SOA is just the edge between the gain of light propagating from wider to narrower and from narrower to wider.

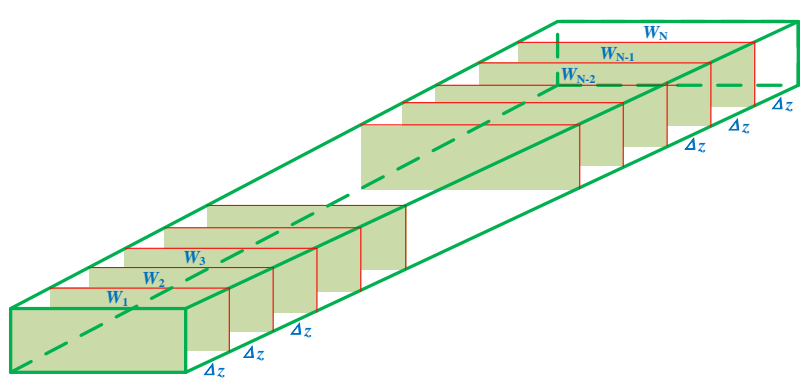

Figure 2. The segmentation model of the non-uniform SOA. 


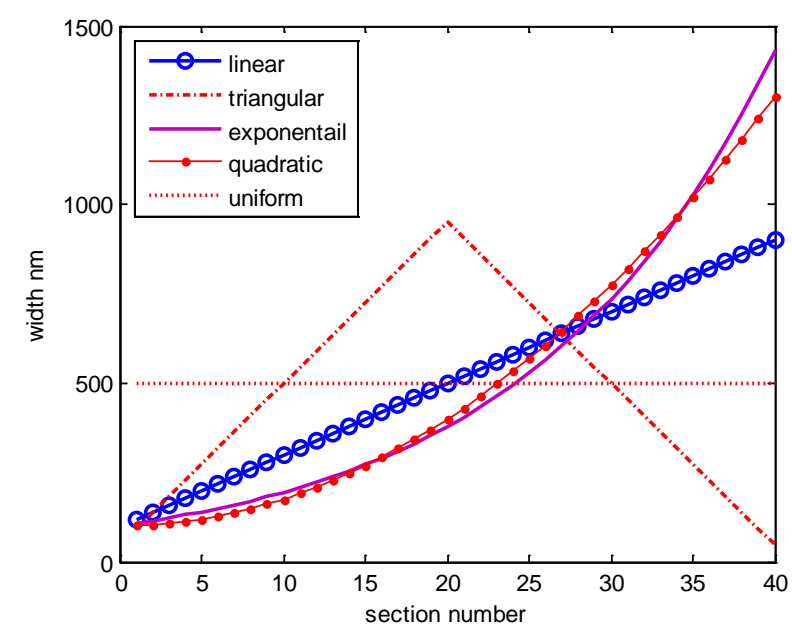

(a) width

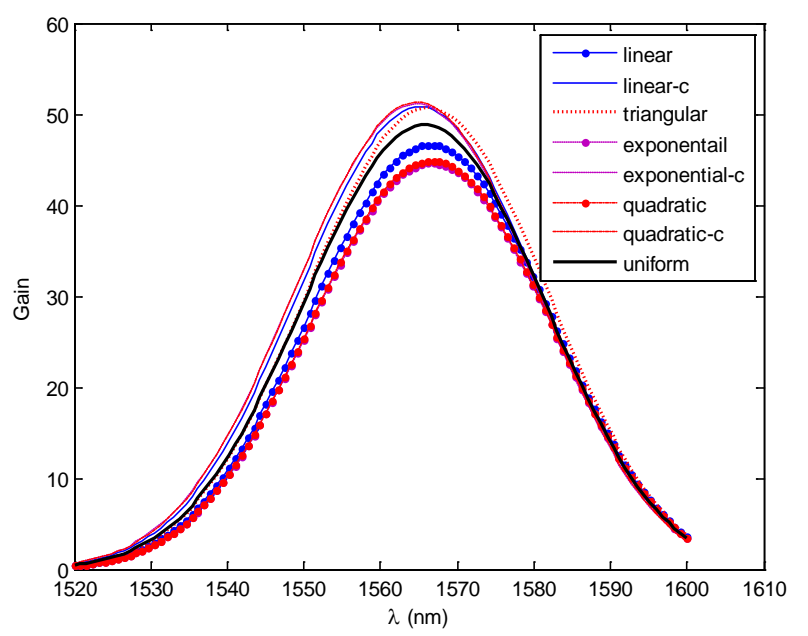

(b) Gain spectra

Figure 3. The width and the gain spectra of the non-uniform SOA for $W_{\text {ave }}=500 \mathrm{~nm}$ and $b=0.2 W_{\text {ave }}$.

The peak gain wavelength shows some dependence on the structure of the non-uniform SOA, and of course it also depends on some other parameters, such as injection current, the temperature. Table 1 shows the parameters in the simulations [13].

\section{Lasing Wavelength of the SOA-RL}

For the ring laser based on the non-uniform SOA, the lasing wavelength $\left(\lambda_{L}\right)$ will be the peak gain wavelength due to the competition in the resonator, so the lasing wavelength heavily depends on the SOA's structure and the injection current. In order to only check the effect of the non-uniform width of the active region of the SOA on the lasing wavelength $\left(\lambda_{L}\right)$, other parameters are set as constant, include the length, the height of the active area, the injection current, the confinement factor, the carrier lifetime, etc.. Figures 4-7 show the lasing wavelength $\left(\lambda_{L}\right)$ of the SOA-RL for the non-uniform width of linear,
Table 1. Some parameters in the simulations.

\begin{tabular}{|c|c|c|}
\hline Parameter & Symbol & Value in SIU \\
\hline Molar fraction of Arsenide in the active region & $\mathrm{y}$ & 0.892 \\
\hline Bandgap energy quadratic coefficient & a & 1.35 \\
\hline Bandgap energy quadratic coefficient & $\mathrm{b}$ & -0.775 \\
\hline Bandgap energy quadratic coefficient & c & 0.149 \\
\hline Effective mass of electron in the CB & $\mathrm{m}_{\mathrm{e}}$ & $4.10 \times 10^{-32}$ \\
\hline Effective mass of heavy hole in the VB & $\mathrm{m}_{\mathrm{hh}}$ & $4.19 \times 10^{-31}$ \\
\hline Effective mass of light hole in the VB & $\mathrm{m}_{\mathrm{lh}}$ & $5.06 \times 10^{-31}$ \\
\hline Auger recombination coefficient & $\mathrm{C}_{\text {aug }}$ & $3.0 \times 10^{-41}$ \\
\hline Leakage recombination coefficient & $D_{\text {leak }}$ & $0.00 \times 10^{48}$ \\
\hline Linear radiative recombination coefficient & $A_{\text {rad }}$ & $1 \times 10^{7}$ \\
\hline $\begin{array}{l}\text { Bimolecular radiative } \\
\text { recombination coefficient }\end{array}$ & $\mathrm{B}_{\mathrm{rad}}$ & $5.6 \times 10^{-16}$ \\
\hline $\begin{array}{l}\text { Linear nonradiative } \\
\text { recombination coefficient due to traps }\end{array}$ & $A_{\text {nrad }}$ & $3.5 \times 10^{8}$ \\
\hline $\begin{array}{l}\text { Bimolecular nonradiative } \\
\text { recombination coefficient }\end{array}$ & $B_{\text {nrad }}$ & $0.00 \times 10^{-16}$ \\
\hline Length & $\mathrm{L}$ & 700 micron \\
\hline Height & d & $400 \mathrm{~nm}$ \\
\hline Light velocity in vacuum & c & $3 \times 10^{8}$ \\
\hline Active region refractive index & $\mathrm{n}_{1}$ & 3.22 \\
\hline Optical confinement factor & $\Gamma$ & 0.45 \\
\hline
\end{tabular}

triangular, exponential and quadratic function with the position.

Figure 4(a) shows the relationships between the lasing wavelength and the linearly non-uniform width for parameters $b_{0} / W_{\text {ave }}$ being $0.2,0.4,0.6$ and 0.8 , the $\lambda_{L}$ of both directional propagating are illustrated together with the uniform SOA. It is interesting that the dependence of the lasing wavelength on the average width is a bit stronger when the light propagates from narrower to wider end than conversely. The lasing wavelength of " $0.8-\mathrm{c}$ " shows almost independent on the average width. In order to check it accurately, Figure 4(b) shows the lasing wavelength for $b_{0} / W_{\text {ave }}$ from 0.7 to 0.92 (spacing 0.02 ) propagating from wider to narrower end. The maximum wavelength difference for " $0.8-\mathrm{c}$ " is less than $6 \mathrm{pm}$ within the average width range from 0.3 to $0.8 \mathrm{mi}-$ crons.

Figure 5(a) shows the relationships between the lasing wavelength and the triangularly non-uniform width for parameters $b_{1} / W_{\text {ave }}$ between 0.1 and 1.8 (spacing 0.1 ). When $b_{1} / W_{\text {ave }}$ is less than 1 , the active area becomes wider from both end to the middle, and becomes narrower from both end to the middle when it is greater than 1 , and uniform when $b_{1} / W_{\text {ave }}=1$. The $\lambda_{L}$ increases with the average width for all cases, the lines are closer for greater $b_{1} / W_{\text {ave }}$ than smaller. It means that the lasing wavelength is stable at a particular average width when the slope is in some range. Figure 5(b) shows the dependence of $\lambda_{L}$ on $b_{1} / W_{\text {ave }}$ for different average width, the maximum wave- 


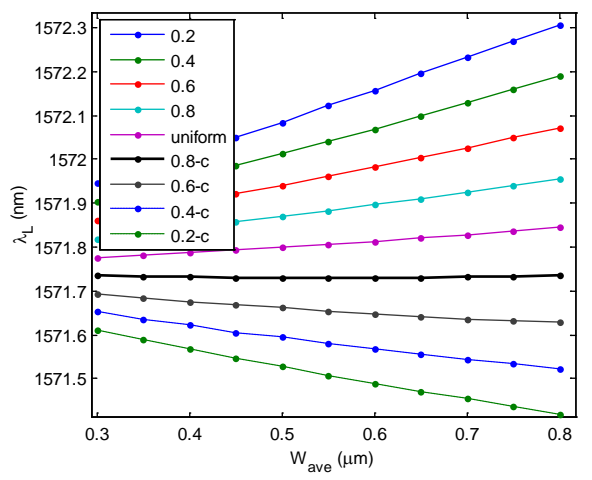

(a)

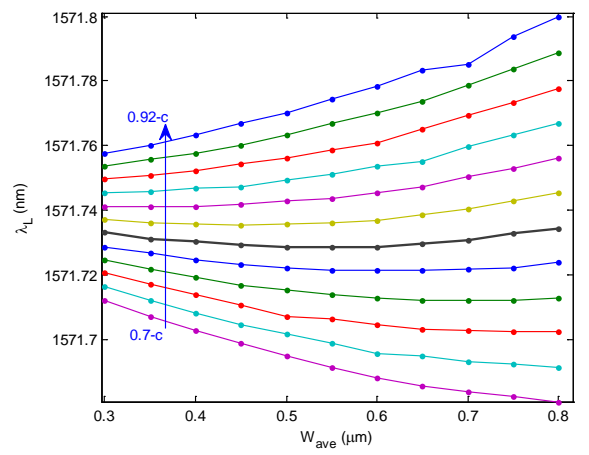

(b)

Figure 4. The lasing wavelength of the SOA-RL for the linear non-uniform width.

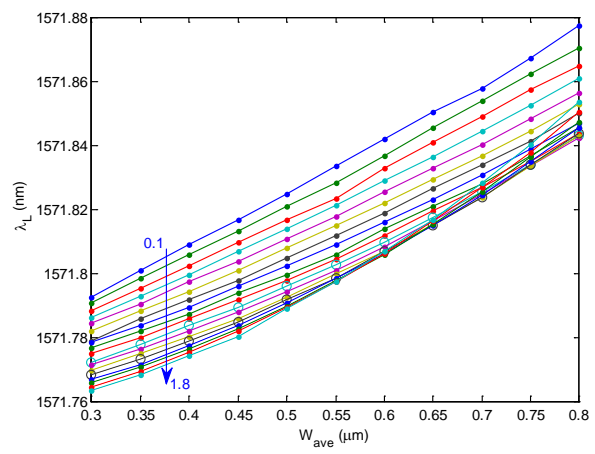

(a)

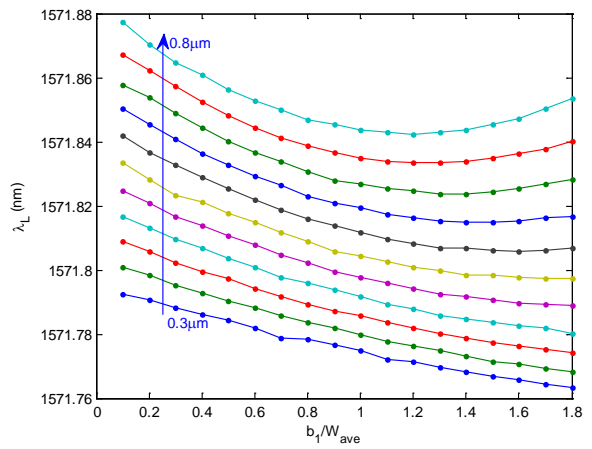

(b)

Figure 5. The lasing wavelength of the SOA-RL for the triangular non-uniform width. length difference is about $7 \mathrm{pm}$ for $W_{\text {ave }}=0.75 \mu \mathrm{m}$ when $b_{1} / W_{\text {ave }}$ is greater than 1 , and about $4 \mathrm{pm}$ for $W_{\text {ave }}=0.55$ $\mu \mathrm{m}$ when $b_{1} / W_{\text {ave }}$ is greater than 1.3 .

Figure 6(a) shows the relationships between the lasing wavelength and the exponentially non-uniform width for parameters $b_{2} / W_{\text {ave }}$ from 0.1 to 0.9 with the spacing 0.1 , the $\lambda_{L}$ of both directional propagating are illustrated together. It is also interesting that the dependence of the lasing wavelength on the average width is a bit stronger when the light propagates from narrower to wider end than conversely. The lasing wavelength of " $0.8-\mathrm{c}$ " shows almost independent on the average width. In order to check it clearly, Figure 6(b) shows the lasing wavelength for $b_{2} / W_{\text {ave }}$ from 0.71 to 0.9 (spacing 0.01 ) propagating from wider to narrower end. The maximum wavelength difference for " $0.8-\mathrm{c}$ " is less than $4.5 \mathrm{pm}$ within the average width range from 0.3 to 0.8 microns.

Figure 7(a) shows the relationships between the lasing wavelength and the quadratically non-uniform width for parameters $b_{3} / W_{\text {ave }}$ from 0.1 to 0.9 with the spacing 0.1 , the $\lambda_{L}$ of both directional propagating are illustrated together. It also shows that the dependence of the lasing wavelength on the average width is stronger when the light propagates from narrower to wider end than conversely. The lasing wavelength of " $0.9-c$ " shows almost independent on the average width. In order to check it

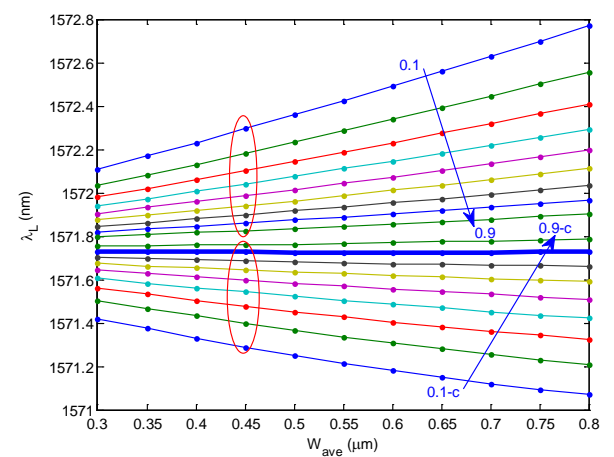

(a)

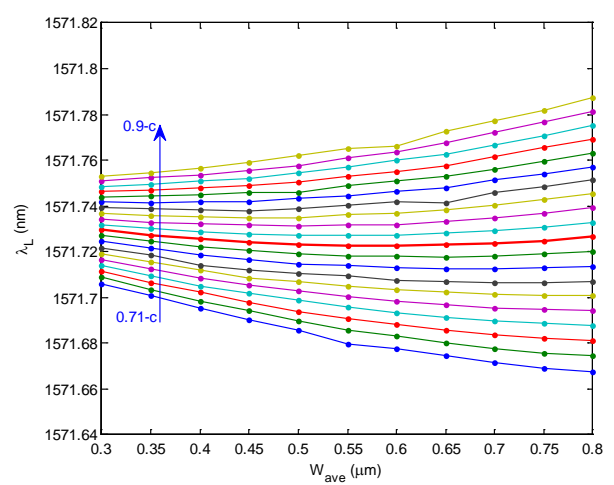

(b)

Figure 6. The lasing wavelength of the SOA-RL for the exponential non-uniform width. 


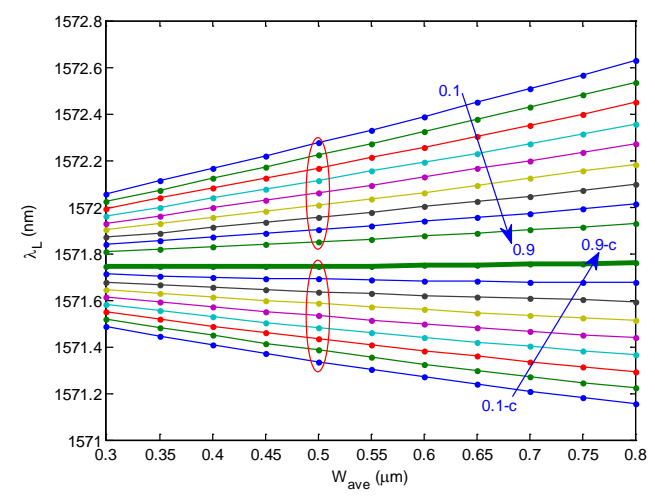

(a)

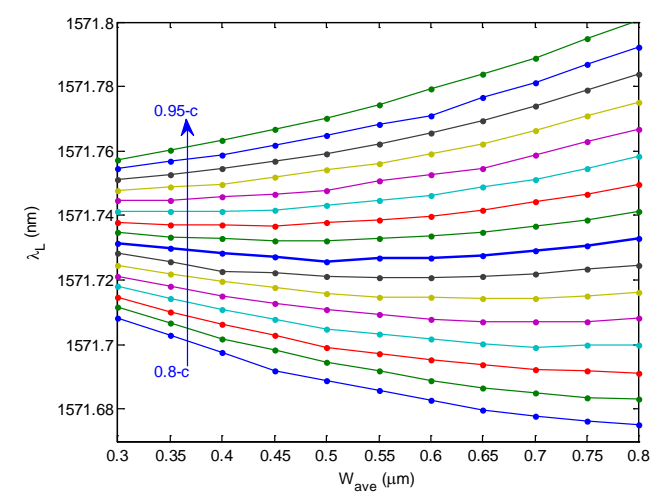

(b)

Figure 7. The lasing wavelength of the SOA-RL for the quadratic non-uniform width.

clearly, Figure 7(b) shows the lasing wavelength for $b_{3} / W_{\text {ave }}$ from 0.8 to 0.95 (spacing 0.01 ) propagating from wider to narrower end. The maximum wavelength difference for " $0.8-\mathrm{c}$ " is less than $6.8 \mathrm{pm}$ within the average width range from 0.3 to 0.8 microns.

\section{Discussion and Conclusion}

The gain property for a few kind of non-uniform SOA is simulated, and the lasing wavelength of the ring cavity laser with the non-uniform SOA is investigated with the dependence on the active area width and the non-uniformity. It shows that the dependence of the lasing wavelength on the average width is stronger when the light propagates from narrower to wider end than conversely. It is supposed that the main reason could be the carrier density distribution along the propagation.

\section{Acknowledgements}

This work was supported in part by the National Natural Science Foundation of China under grant 61077048, Beijing Natural Science Foundation under grant 4132035, Specialized Research Fund for the Doctoral Program of Higher Education under grant 20120009110032, and by Beijing Jiaotong University under grant 2012JBM103.

\section{REFERENCES}

[1] Y. Liu, M. T. Hill, N. Calabretta, H. dewaardt, G. D. Khoe, Fellow and H. J. S. Dorren, "Three-State All-Optical Memory Based on Coupled ring Lasers,” IEEE Photon. Technol. Lett., Vol. 15, No. 10, 2003, pp. 1461-1464. http://dx.doi.org/10.1109/LPT.2003.818221

[2] Q. F. Xu and M. Y. Yao, "Theoretical Analyses on ShortTerm Stability of Semiconductor Fiber Ring Lasers," IEEE Journal of Quantum Electron, Vol. 39, No. 10, 2003, pp. 960-965.

[3] H. X. Chen, "Multiwavelength Fiber Ring Lasing by Use of a Semiconductor Optical Amplifier," Optics Letters, Vol. 30, No. 6, 2005, pp. 619-621. http://dx.doi.org/10.1364/OL.30.000619

[4] L. T. Wang, N. A. Fang, Y. Wang and Z. M. Huang, "DualChannel PolSK Optical Transmissions Using SOA-Based All-Optical Polarization Modulations," Acta Optica Sinica, Vol. 29, No. 1, 2009, pp. 138-144. http://dx.doi.org/10.3788/AOS20092901.0138

[5] S. R. Chinn, E. A. Swanson, and J. G. Fujimoto, “Optical Coherence Tomography Using a Frequency Tunable Optical Source,” Optics Letters, Vol. 22, No. 5, 1997, pp. 340-342. http://dx.doi.org/10.1364/OL.22.000340

[6] J. Leuthold and M. Kauer, "Power Equalisation and Signal Regeneration with Delay Interferometer All-Optical Wavelength Converters," Electronics Letters, Vol. 38, No. 24, 2002, pp. 1567-1568. http://dx.doi.org/10.1049/el:20021077

[7] C. Habib, V. Baby, L. R. Chen, A. Delisle-Simard and S. LaRoehelle, "All-Optical Swapping of Spectral Amplitude Code Labels Using Nonlinear Media And Semiconductor Fiber Ring Lasers," IEEE Journal of Selected Topics in Quantum Electronics, Vol. 14, No. 3, 2008, pp. 879-888. http://dx.doi.org/10.1109/JSTQE.2008.918047

[8] P. Wei, "Fiber Ring Cavity Semiconductor Laser of Research,” Ph.D. Thesis, Southwest Jiaotong University, Chengdu, 2000.

[9] M. Schell and E. Schol, "Time-Dependent Simulation of a Semiconductor Laser Amplifier: Pulse Compression in a Ring Configuration and Dynamic Optical Bistability,” IEEE Journal of Quantum Electronics, Vol. 26, No. 6, 1990, pp. 1005-1013.

http://dx.doi.org/10.1109/3.108096

[10] L. F. Stokes, M. Chodorow and H. J. Shaw, "All-SingleMode Fiber Resonator,” Optics Letters, Vol. 7, No. 6, 1982, pp. 288-290. http://dx.doi.org/10.1364/OL.7.000288

[11] H. Taleb, K. Abedi and S. Golmohammadi, "Operation of Quantum-Dot Semiconductor Optical Amplifiers under Nonuniform Current Injection,” Applied Optics, Vol. 50, No. 5, 2011, pp. 608-617. http://dx.doi.org/10.1364/AO.50.000608

[12] M. J. Connelly, "Wideband Semiconductor Optical Amplifier Steady-State Numerical Model,” IEEE Journal of Quantum Electronics, Vol. 37, No. 3, 2001, pp. 439-448. http://dx.doi.org/10.1109/3.910455 